\title{
Propuesta de una metodología de evaluación del aprendizaje basada en las TIC
}

\section{M.D.ReyesTolosa ${ }^{\mathrm{a}}$, O. Sahuquillo ${ }^{\mathrm{a}}$}

${ }^{a}$ Departamento de Ingeniería Mecánica y de Materiales, Universitat Politècnica de València, Camino de Vera s/n, 46022, Valencia. mareto@upvnet.upv.es

\begin{abstract}
Is vital for teachers to assess accurately the continuous learning of students, obtaining of this assessment is not just a rating of each one of the students, if not also monitor the student's learning, and can reinforce those topics that are necessary.

To this end, a series of questions, each of the topics of theoretical, using an online tool, have been implemented specifically SOCRATIVE, which is a tool that incorporates the use of technology and is made available from the learning. Methodology highlights because it is an active and motivational methodology with the advantage of offering the results instantly and above all enable new possibilities in the learning process.

In addition, at end of course, it is planned a survey of evaluation of the methodology in order to optimize its use in subsequent courses.
\end{abstract}

Keywords: evaluation of learning, online, methodology tool active

\begin{abstract}
Resumen
Es de vital importancia para los docentes poder evaluar de forma precisa el aprendizaje continuo de los alumnos, obteniendo de esta evaluación no solo una calificación de cada uno de los alumnos, sino también un seguimiento del aprendizaje del alumno, pudiendo reforzar aquellos temas que sean necesarios.
\end{abstract}

Para ello, se han implementado una serie de preguntas, correspondientes a cada uno de los temas teóricos, empleando una herramienta online, concretamente SOCRATIVE, siendo esta una herramienta en la que se incorpora el uso de la tecnología y se pone a disposición del aprendizaje. La metodología destaca por tratarse de una metodología activa y motivadora con la ventaja de ofrecer los resultados instantáneamente y sobretodo permitir nuevas posibilidades en el proceso de aprendizaje.

Además, a final de curso, se ha previsto una encuesta de evaluación de la metodología con el fin de optimizar en cursos posteriores su uso.

Palabras clave: evaluación del aprendizaje, herramienta online, metodología activa. 


\section{Introducción}

Como es sabido, el nuevo marco docente dibujado por la convergencia hacia el Espacio Europeo de Educación Superior (EEES) supone una reformulación de las metodologías docentes, que han de estar basada en el aprendizaje del alumno y no solo en la enseñanza. Es por ello que en la docencia superior universitaria se desarrollan en los últimos años nuevas formas de llegar al alumno, empleando para ello aquellas herramientas con las que están familiarizados y que despiertan su interés.

De hecho, los docentes universitarios nos encontramos ante un alumnado diferente, debido precisamente a su generación, la llamada generación Z, aquellos nacidos a partir de 2001 hasta la actualidad, los cuales se caracterizan por las siguientes características mostradas en la tabla 1.

Tabla 1. Generación Z

\begin{tabular}{|c|c|c|c|c|}
\hline Generación & $\begin{array}{l}\text { Periodo de } \\
\text { nacimiento }\end{array}$ & Contexto nacional & $\begin{array}{c}\text { Herramientas } \\
\text { educativas }\end{array}$ & $\begin{array}{c}\text { Modelo pedagógico- } \\
\text { educativo }\end{array}$ \\
\hline $\begin{array}{c}\text { Nativos } \\
\text { digitales o } \\
\text { generación Z }\end{array}$ & $\begin{array}{l}\text { 2001- } \\
\text { Actualidad }\end{array}$ & $\begin{array}{l}\text { Avances importantes en } \\
\text { tecnología } \\
\text { computacional (tablets, } \\
\text { impresión 3D, etc.) } \\
\text { telefonía móvil, redes } \\
\text { sociales, economía del } \\
\text { conocimiento, } \\
\text { innovación de productos } \\
\text { y servicios globalizados }\end{array}$ & $\begin{array}{c}\text { Herramientas } \\
\text { tecnológicas } \\
\text { educativas desde nivel } \\
\text { escolar como las } \\
\text { pizarras electrónicas, } \\
\text { proyecciones en el } \\
\text { aula, etc. }\end{array}$ & $\begin{array}{c}\text { Competencias bajo el } \\
\text { enfoque socio- } \\
\text { formativo, el profesor es } \\
\text { un facilitador y el } \\
\text { alumno una persona de } \\
\text { aprendizaje significativo } \\
\text { autónomo y responsable } \\
\text { que se enfrenta a } \\
\text { problemas críticos }\end{array}$ \\
\hline
\end{tabular}

En este contexto, la responsabilidad del docente ya no sólo es tener el conocimiento de la asignatura y transmitirlo, si no ahora está la responsabilidad de aprender a utilizar las herramientas tecnológicas actuales (TIC), enseñar los estudiantes a encontrar la información correcta en sitios web fiables, analizar y sintetizar esta información y convertirla en competencias para su desarrollo (Echevarría, 2017).

Del mismo modo que las herramientas de docencia cambian de acuerdo a la generación a la que el docente se expone, han de cambiar también los métodos de evaluación. La evaluación en el ámbito académico, comparte la función formativa y certificadora (Villardón 2006). La dimensión formativa de la evaluación ha de complementarse con la función meramente certificadora (López, 2009). La evaluación así entendida ha de centrarse más en el alumno y su aprendizaje (Goñi 2005) que en la enseñanza del profesor y, si el alumno ha de ser el protagonista de su propio aprendizaje, es lógico que este participe en su proceso de evaluación (Arribas, 2012), donde los métodos basados en la coevaluación cada vez más van teniendo más relevancia.

(cc) EY-NC-ND 2018, Universitat Politècnica de València 
Así pues, parece evidente el enorme potencial que puede tener el uso de herramientas TIC en el aprendizaje de los alumnos de generación $\mathrm{Z}$, de forma que se implanta a su vez, una metodología activa por medio de la autoevaluación, con un elevado factor motivador para los alumnos debido al uso de las mismas.

\section{Objetivos}

El principal objetivo se basa en evaluar parte del aprendizaje del alumno durante el curso mediante el uso de nuevas tecnologías (TIC) con herramientas online con un doble enfoque: como herramienta de evaluación desde el punto de vista del docente y como autoevaluación continua para el alumno.

Se plantea el desarrollo de un sistema de evaluación atractivo para el alumno, conciso y con retroalimentación inmediata, de forma que tanto el docente como el alumno puedan evaluar la formación continua, el aprendizaje y el seguimiento de la materia. Constituye así una prueba experimental cuyo objetivo radica en la evaluación alternativa a los portafolios escritos manualmente empleados en la evaluación actual.

\section{Desarrollo de la innovación}

El desarrollo de la innovación surge ante la necesidad del docente de poder evaluar de forma continua al alumno fundamentalmente en grupo medianos o grandes, ofreciéndole al mismo mediante esta evaluación una autoevaluación a lo largo del curso, despertando así en el alumno la auto superación y a la vez, preparándole para la evaluación final de la asignatura.

Se decidió realizar una evaluación de manera continua a lo largo del cuatrimestre, de forma que tras la exposición de uno o varios temas teóricos y su posterior estudio práctico en el aula por parte del profesor, el alumno debería contestar una serie de preguntas de una dificultad media-alta. De manera que el alumno, se enfrenta a preguntas "tipo examen" y puede autoevaluar su nivel de aprendizaje y la eficacia de su estudio. A su vez, el docente dispone de una valoración del esfuerzo continuo de cada uno de los alumnos, sabiendo donde reforzar sus explicaciones, disponer registro de su evolución y disponer de datos fiables y objetivos para su evaluación global.

El método propuesto desarrollado para la evaluación continua tiene las siguientes características principales, de forma que resulte lo más atractivo:

- Uso de nuevas tecnologías TIC

- Atractiva para el alumno, técnica de gamificación (aprender jugando)

- Facilidad de uso en el aula y accesible a un gran número de alumnos

- Retroalimentación inmediata

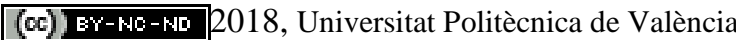

Congreso In-Red (2018) 
Se decidió, por tanto, el uso de una APP comercial (tecnología TIC) que el alumno pudiera instalar de forma gratuita en su Smartphone o en pc propio o del aula, así como accediendo directamente a la web con la intención de ser atractiva para el alumno, y de fácil uso en el aula. Concretamente se eligió la aplicación SOCRATIVE entre otras, por su facilidad de uso y la capacidad para captar la atención del alumnado gracias al amplio abanico de posibilidades interactivas que ofrece.

Se trata de una herramienta gratuita online que actúa como un sistema de respuesta inteligente con el que el docente puede lanzar varios tipos de preguntas, quizzes, juegos, a los que los alumnos pueden responder en tiempo real desde sus dispositivos. Una vez acabada la serie de preguntas el profesor puede ver los informes de resultados como una hoja de cálculo y puede emplearlo para realizar la evaluación.

La metodología de evaluación que se ha implementado en SOCRATIVE, consiste en la formulación de preguntas con 4 respuestas posibles a modo de quizz.

En tiempo real, el profesor puede ver los alumnos que se han unido al ejercicio, los cuales deben identificarse con su nombre completo, para poder exportar posteriormente los resultados identificando al alumno. Cada uno de estos alumnos, contestará a las preguntas, bien con su Smartphone o pc, en la Fig. 1 se muestra un ejemplo de la visualización en el dispositivo. El orden de las preguntas y respuestas posibles en cada una de ellas es aleatorio para cada alumno, de manera que se reduce el riesgo de copiado.

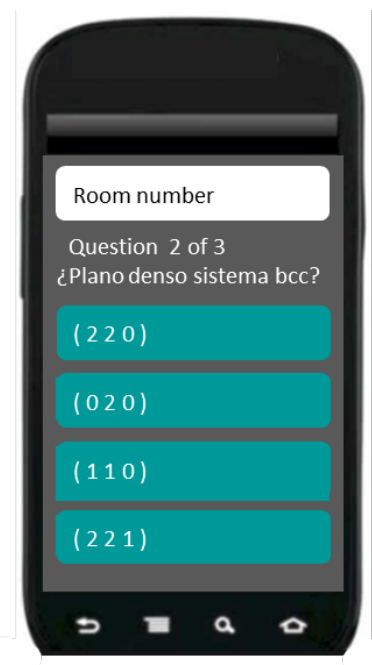

Fig. 1 Ilustración de visualización de la pregunta en un smartphone

A medida, que los alumnos van respondiendo a las preguntas se puede realizar un seguimiento de aquellos que han contestado y de los que todavía faltan por hacerlo. Durante la realización del ejercicio sólo se visualiza el progreso de las cuestiones planteadas sin mostrar las respuestas individuales de cada alumno, como muestra la Fig.2.

(cc) BY-NC-ND 2018, Universitat Politècnica de València

Congreso IN-RED (2018) 


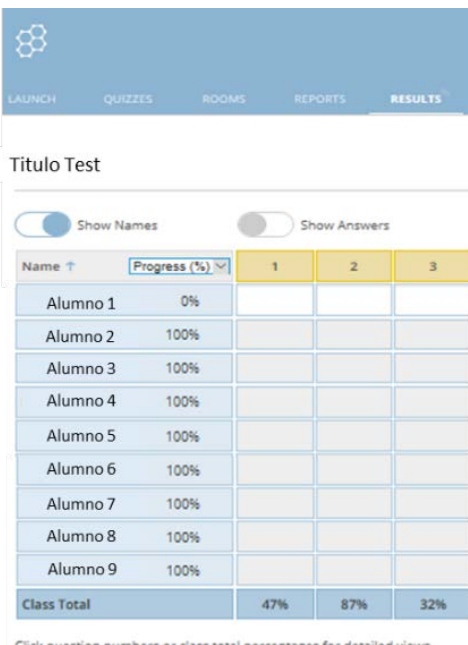

Fig. 2 Visualización del progreso del ejercicio

Finalmente, el número de respuestas correctas que conteste el alumno respecto al total planteadas se tendrá en cuenta en la calificación correspondiente al portafolio del alumno, constituyendo estos un punto sobre diez en la calificación global de la asignatura en la que se ha implementado como experiencia piloto, siendo esta la que se expresa en base 10:

NOT $A=0.7 \cdot$ Examen teoría y problemas $+0.2 \cdot$ Laboratorio $+0.1 \cdot$ Portafolio

De ese mismo modo, la metodología también supone incentivar la motivación y la competitividad, con una técnica basada en la gamificaicón, donde los alumnos se espera que se esfuercen en el aprendizaje continuo de la asignatura para intentar conseguir el mayor número de respuestas correctas fomentando el espíritu de superación, pudiendo además comparar con las obtenidas por sus compañeros.

Con la intención de poder evaluar la metodología empleada se propone una encuesta final a los alumnos (Barrado, 1999), de forma que se tenga una evaluación del método. La encuesta desarrollada se presenta en la Tabla 2. 
Tabla 2.-Encuesta de satisfacción con la evaluación continua y TIC empleada

\begin{tabular}{|l|c|}
\hline \multicolumn{1}{|c|}{ PREGUNTA } & RESPUESTA \\
\hline El método de evaluación continua está claro desde el inicio de curso & SI \\
\hline El método de evaluación continua es justo & SI \\
\hline La metodología TIC empleada me resulta fácil y práctica & SI NO \\
\hline $\begin{array}{l}\text { Las preguntas realizadas en Socrative me han servido para detectar los } \\
\text { aspectos que debo reforzar }\end{array}$ & SI NO \\
\hline $\begin{array}{l}\text { La evaluación continua realizada con esta metodología me ha ayudado en } \\
\text { el aprendizaje continuo de la asignatura }\end{array}$ & SI \\
\hline $\begin{array}{l}\text { ¿Consideras la metodología empleada motivadora en el aprendizaje de la } \\
\text { asignatura? }\end{array}$ & SI \\
\hline $\begin{array}{l}\text { A pesar de sus limitaciones, consideras que la metodología utilizada } \\
\text { debería de seguir utilizándose incluso en otras asignaturas }\end{array}$ & SI \\
\hline
\end{tabular}

\section{Resultados}

La prueba piloto del nuevo método de evaluación continua se está desarrollando en la actualidad en el Grado de Ingeniería Aeroespacial, en la asignatura de Ciencia de Materiales del primer curso de la E.T.S. Ingeniería del Diseño de la Universitat Politècnica de València, concretamente en uno de los grupos de teoría que cuenta aproximadamente con 80 alumnos matriculados en el presente curso. Dicha asignatura se imparte en el segundo cuatrimestre y por lo tanto actualmente se encuentra en desarrollo y tan solo se tienen resultados tras mes y medio de evaluación.

Los resultados que se muestras a continuación se corresponden con la primera sesión de preguntas formuladas a través de SOCRATIVE.

Una vez, se ha realizado el quizz en el aula, el profesor puede generar una hoja de cálculo con los resultados obtenidos, de forma que resulta muy fácil y práctico extraer conclusiones. La siguiente imagen (Fig.3) muestra un ejemplo de cómo se muestran esos resultados. 


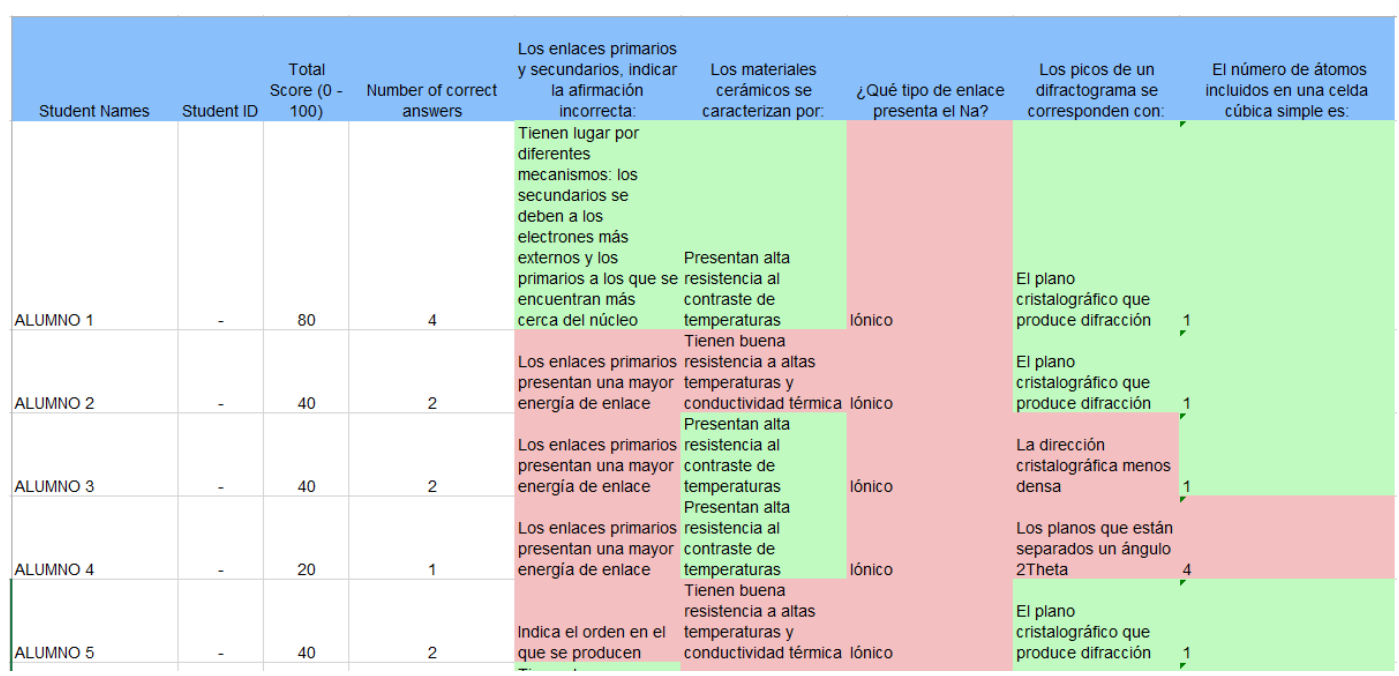

Fig.3 Visualización de resultados en excel

Como puede verse en la Fig.3. el programa SOCRATIVE nos genera una hoja de cálculo de resultados en el que se puede ver para cada alumno:

- Puntuación obtenida

- Numero de respuestas correctas

- Respuesta marcada por el alumno: correcta (verde) o incorrecta (rojo).

Resulta por tanto, muy práctico el feedback prácticamente instantáneo que el profesor recibe y con el que puede detectar que parte del temario es aquella en la que más dificultades están encontrando los alumnos, y poder así reforzarla. Al igual, que de forma directa se obtiene una calificación del alumno para el ejercicio desarrollado. Así mismo el alumno puede antes de la evaluación a través de parciales detectar sus deficiencias y poner medios para superarlas.

Además se pretende realizar un análisis para cada uno de los ejercicios, de forma que el docente pueda autoevaluar y discriminar la efectividad de las preguntas formuladas. Es decir, se puede determinar que preguntas de las formuladas en los ejercicios discriminan el nivel de esfuerzo o aprendizaje de los alumnos, diferenciándose preguntas:

- Muy buenas: aquellas que solo contestan bien los alumnos que contestan bien a más del $80 \%$ de las preguntas propuestas.

- Buenas: aquellas que solo contestan correctamente los alumnos que contestan bien entre el 60 y $80 \%$ de las preguntas formuladas.

- Regulares: preguntas que contestan bien los alumnos que saben la respuesta correcta de al menos el 50\% de las preguntas

- Malas: aquellas que contestan bien todos o prácticamente todos los alumnos. 
Hasta el momento, tan solo se tienen datos de un primer ejercicio, y de él se pueden extraer los siguientes resultados que se exponen:

- En un primer ejercicio de 5 preguntas con 4 respuestas posibles por cuestión, el porcentaje de alumnos que ha contestado bien a 3 o más preguntas (calificación de aprobado o superior) constituye un 59\%. Por tanto, puede decirse que aproximadamente el 60\% de los alumnos mantiene un estudio activo y continuo de la asignatura, y que además la metodología docente y de estudio por parte del alumno resulta efectiva. Tal y como puede verse en la figura 4.

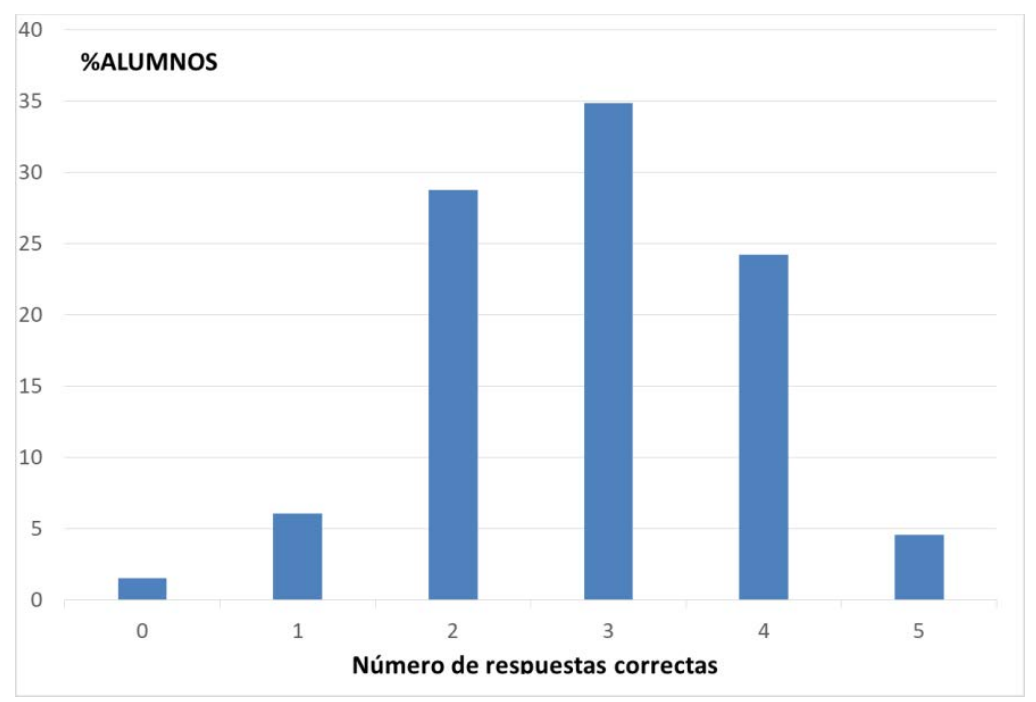

Fig.4 Distribución de preguntas correctamente contestadas.

- Contabilizando el número de alumnos que ha contestado bien cada una de las peguntas, se puede determinar la eficiencia de dicha pregunta, en el caso de aplicación, se ha obtenido:

Tabla 2.-Calidad de las preguntas formuladas en función del porcentaje de acierto

\begin{tabular}{|l|l|l|l|l|l|}
\hline & PREG.1 & PREG.2 & PREG.3 & PREG 4. & PREG 5. \\
\hline \% ACIERTOS & $25.75 \%$ & $33.33 \%$ & $59 \%$ & $59 \%$ & $96.97 \%$ \\
\hline
\end{tabular}

Se extrae, por tanto que si bien la pregunta 1 y 2 son altamente discriminatorias entre aquellos alumnos que han estudiado de los que no, y que por tanto pueden clasificarse como muy buena y buena respectivamente, no ocurre lo mismo con las preguntas 3, 4 y 5, siendo esta última de categoría mala pues el 95\% de los alumnos la contesta acertadamente sin discriminar entre aquellos que han estudiado y de los que no.

(cc) BY-NC-ND 2018, Universitat Politècnica de València 
Ahondando más en el análisis de los resultados para ver la efectividad del método, se han realizado los cálculos de los "índices de dificultad y discriminación de las preguntas”(Morales, 2009), de forma que los docentes tengamos la información necesaria para evaluar nuestras propias preguntas.

Dichos índices, se calculan con el 25\% de los alumnos que han obtenido las notas más altas y el $25 \%$ de los alumnos que han obtenido una puntuación total más baja. El número de sujetos en ambos grupos es el mismo y sólo se analiza el 50\% de alumnos (se prescinde del $50 \%$ central).

Se definen:

- Índice de dificultad: Indica la proporción de aciertos en la muestra de alumnos que estamos utilizando. Un índice mayor indica que la pregunta es más fácil.

$$
D_{f}=\frac{A S+A I}{N+N}
$$

Siendo:

- AS: Número de acertantes en el grupo superior con la puntuación total más alta.

- $\quad$ AI: Número de acertantes en el grupo inferior con la puntuación total más baja.

- N: Número de sujetos analizados en uno de los dos grupos (ambos grupos tienen idéntico número de sujetos)

En nuestro caso para cada una de las preguntas realizadas:

Tabla 3.-Indice de dificultad para cada una de las preguntas formuladas

\begin{tabular}{|c|c|c|c|c|}
\hline & AS & AI & N & Df \\
\hline P1 & 16 & 0 & 16 & $\mathbf{0 , 5}$ \\
\hline P2 & 16 & 0 & 16 & $\mathbf{0 , 5}$ \\
\hline P3 & 16 & 0 & 16 & $\mathbf{0 , 5}$ \\
\hline P4 & 16 & 0 & 16 & $\mathbf{0 , 5}$ \\
\hline P5 & 16 & 14 & 16 & $\mathbf{0 , 9 4}$ \\
\hline
\end{tabular}

Se corrobora mediante el análisis de este índice que la pregunta 5 es claramente fácil y no discriminatoria, ya que ha tenido un $949 \%$ de aciertos.

- Índice de discriminación: Consiste en la diferencia entre dos proporciones, proporción de aciertos en el grupo superior (AS/N) menos la proporción de aciertos en el grupo inferior $(\mathrm{AI} / \mathrm{N})$. Expresa, por tanto hasta qué punto una pregunta es capaz de discriminar.

En nuestro caso:

(cc) EY-NC-ND 2018, Universitat Politècnica de València 


$$
D_{c}=\frac{A S-A I}{N}
$$

Tabla 4.-Indice de discriminación para cada una de las preguntas formuladas

\begin{tabular}{|c|c|c|c|c|}
\hline & AS & AI & N & Dc \\
\hline P1 & 16 & 0 & 16 & $\mathbf{1}$ \\
\hline P2 & 16 & 0 & 16 & $\mathbf{1}$ \\
\hline P3 & 16 & 0 & 16 & $\mathbf{1}$ \\
\hline P4 & 16 & 0 & 16 & $\mathbf{1}$ \\
\hline P5 & 16 & 14 & 16 & $\mathbf{0 , 1 2 5}$ \\
\hline
\end{tabular}

Analizando los resultados del índice obtenido, se puede determinar que las 4 primeras preguntas, son discriminatorias ya que únicamente las aciertan los del grupo superior, mientras que no hay ninguna respuesta correcta en el grupo inferior. Haciendo así que el índice alcance su valor máximo.

Al contrario ocurre con la pregunta número 5, con un índice de 0.125 , lo que significa, que tanto alumnos del grupo inferior y superior contestan acertadamente a la pregunta, y por lo tanto no discrimina, lo que significa que es una pregunta muy fácil y conviene que sea revisada.

\section{Conclusiones}

En primera instancia y a falta de la encuesta de satisfacción, en la primera sesión con los alumnos la han acogido con satisfacción basándonos en la percepción del docente. Además, los alumnos agradecen el poder practicar este tipo de pruebas objetivas antes de la evaluación escrita, ya que por lo general tienden a no sentirse muy seguros antes los exámenes tipo test, y de esta forma pueden practicar y conocer la forma de preguntar del docente, ganando así seguridad.

El uso de las TIC predispone a los alumnos con una actitud receptiva hacia el aprendizaje, por el uso del smartphone o el pc, así como introducir la componente juego y competición a la hora de intentar conseguir el mejor resultado posible y que además formará parte de su evaluación final.

La metodología y la herramienta son de fácil uso tanto para el profesor como para el alumno, como también lo es la interpretación de los resultados derivados de este acto de evaluación.

En cuanto al feedback de la metodología empleada al alumno le reporta información por medio de su autoevaluación durante todo el periodo lectivo y el profesor recibe la

(cc) EY-NC-ND 2018, Universitat Politècnica de València 
retroalimentación de aquellos puntos en los que los alumnos están encontrando más dificultades y por tanto puede reforzarlos.

A priori y con sólo los primeros resultados obtenidos, parece ser una posible alternativa los portafolios escritos manualmente dado que es posible emplearla en grupos de gran tamaño además de resultar atractiva al alumnado y reportarle información sobre su progreso y servir de retroalimentación y como herramienta de evaluación al profesor.

Mediante el uso de este tipo de evaluación, y realizando el análisis del mismo,tanto el alumno como el docente adquieren un aprendizaje continuo.

$>$ El alumno es capaz de evaluar sus conocimientos adquiridos y reforzar aquellos puntos más débiles, además de enfrentarse a una evaluación de tipo objetiva que a priori, tanto les cuesta.

$>$ El docente, es capaz de ver que partes del temario han quedado más claras y cuales menos, pudiendo reforzar en clase estos puntos. Además mediante el análisis de las preguntas formuladas es capaz no solo de identificar el nivel medio del aula, sino también de formular preguntas que garanticen la discriminación de conocimientos entre alumos manteniendo el nivel de dificultad adecuado en cada una de las preguntas que se formulen.

\section{Referencias}

Arribas, J.M. (2012). "El rendimiento académico en función del sistema de evaluación empleado" Revista Electrónica de Investigación y Evaluación educativa.

https://www.uv.es/RELIEVE/v18n1/RELIEVEv18n1_3.htm [Consulta: 22 de marzo de 2018]

Barrado, C., Usemos las encuestas a los alumnospara mejorar nuestra docencia. ReportUPC-DAC1999-70, Departamentd'Arquitectura de Computadors, UniversidadPolitécnica de Cataluña, 1999.

<ftp://ftp.ac.upc.es/pub/reports/DAC/1999/UPC-DAC-1999-70.ps.Z> [Consulta: 22 de marzo de 2018]

Echevarría Echevarría, S.T (2017). "Una formación docente acorde a los cambios generacionales, tecnológicos e innovadores en los programas de postgrado nacionales” en Temas de Ciencia y Tecnología., vol 21, número 63, p 33-38.

Goñi, J.M. (2005). El espacio europeo de educación superior, un reto para la universidad. Barcelona: Ed. Octaedro.

López, V. (2009). “La evaluación formativa y compartida en docencia universitaria: propuestas, técnicas, instrumentos y experiencias”. Madrid: Narcea.

Villardón, L. (2006). "Evaluación del aprendizaje para promover el desarrollo de competencias". Educatio. Siglo XXI, volumen 24, p. 15-35.

Morales, P. (2009). “Análisis de ítems en las pruebas objetivas”. Universidad Pontífica de Comillas, Facultad de Ciencias Humanas y Sociales.

(cc) EY-Nc-ND 2018, Universitat Politècnica de València

Congreso In-Red (2018) 